
\title{
25 Research Square \\ Self-blame regret, fear of COVID-19 and mental health during post-peak pandemic
}

Hacer Belen ( $\boldsymbol{D}$ hacerbelen@uludag.edu.tr)

Bursa Uludag University https://orcid.org/0000-0001-9065-3504

\section{Research Article}

Keywords: Self-blame regret, fear of COVID-19, perceived stress, depression, serial mediation

Posted Date: August 11th, 2020

DOI: https://doi.org/10.21203/rs.3.rs-56485/v1

License: (c) (i) This work is licensed under a Creative Commons Attribution 4.0 International License.

Read Full License

Version of Record: A version of this preprint was published at International Journal of Psychology and Educational Studies on October 31st, 2021. See the published version at https://doi.org/10.52380/ijpes.2021.8.4.447. 


\section{Abstract}

The novel Coronavirus pandemic caused strong negative emotions including fear, and stress and impacted in mental health of individuals worldwide. One of the emotions linked with mental health and infectious disease is self-blame regret. Thus, current study investigated the role of fear of COVID-19 and perceived stress in the relationship between self-blame regret and depression. A community sample of 352 individuals in Turkey (71 \% female and $29 \%$ males), ranged between in age18 and $63(\mathrm{M}=$ 28.90 \pm 8.90 ), completed fear of COVID-19 (FCV-19S), perceived stress (PSS-10), DASS-21 scales and responded to one item concerning the self-blame regret. Results demonstrated that self-blame regret is positively correlated with fear of COVID-19, perceived stress and depressive symptoms. Moreover, serial multiple mediation analyses demonstrated that both fear of COVID-19 and perceived stress mediated in the relationship between self-blame regret and depression. Findings and implications are discussed.

\section{Introduction}

December 2019 witnessed the onset of a "once-in-a-century pandemic" that impacted the individuals worldwide with a case fatality rate similar to 1918 influenza (Gates, 2020). The impact of the disease (COVID-19) is so severe that, as of 13 th of July, over 12 million infected cases were declared with 568.000 death tolls worldwide (European Centre for Disease Prevention and Control, 2020).

Considering the fast transmission of the virus between humans, high mortality rates and strict prevention measures including travel bans, home confinements, social distancing, compulsory face mask wear, school suspensions, this time of global crisis is characterized with strong negative emotions and deteriorated mental health. Several studies reported the heightened negative affectivity symptoms due to the pandemic (Limcaoco et al., 2020) and documented that COVID-19 pandemic exacerbated mental health issues including depression across the world (Bäuerle et al., 2020; Choi, Hui \& Wan, 2020; Nickell et al., 2004; Tsang, Scudds, \& Chan, 2004).

One of the common responses to adversity and life threatening situations is surely stress. Stress arises when an individual perceives that environmental challenges are beyond individual's capacity to cope with (Cohen, Janicki-Deverts \& Miller, 2007). Brooks and colleagues (2020) suggested that COVID-19 pandemic and associated quarantines call forth substantial and long-lasting psychological impact including increased stress. As such, previous research concerning the current pandemic revealed heightened stress among individuals due to fear of infection, transmitting the virus to the loved ones, lack of information and boredom, and loss of social communication (Zandifar \& Badrfam, 2020; Zhang et al., 2020). In support, several studies documented that perceived stress is an important concept to investigate during the pandemic (Du et al., 2020; McAlonan et al., 2007; Mo et al., 2020; Preti et al., 2020) and might be impactful on developing depression symptoms (e.g., Gotlib et al., 2020; Montano \& Acebes, 2020). 
Similar to previous epidemics, COVID-19 pandemic caused strong negative emotions among individuals. Several studies reported greater levels of frustration and boredom (Zhang et al., 2020), increased anxiety and depression (e.g., Nickell et al., 2004; Tsang, Scudds, \&Chan, 2004), heightened fear, and particularly elevated levels of fear of COVID-19 (e.g., Belen, 2020; Reznik et al., 2020). Research regarding the current pandemic highlighted that fear of COVID-19 is associated with critical aspects of mental health (Harper et al., 2020; Pakpour \& Griffiths, 2020; Satici et al., 2020). Thus, as a vital emotion during such a pandemic, exploring this is certainly worth studying.

\section{Self-Blame Regret and Mental Health}

As noted, strong negative emotions exacerbates the mental health issues. Research regarding the emotions and mental health in the context of infectious diseases is still scarce. Yet, one of the researched emotions linked with mental health during the pandemic is regret (Limcaoco et al., 2020). Regret is an emotional experience elicited when an individual is unsatisfied with the acquired outcome among the possibility of better options (Shepherd \& O'Carroll, 2014). Despite the frequency of experiencing anxiety outweighs the regret in daily life, the negative influence of regret is far greater than that of anxiety in terms of mental health (Brewer, DeFrank \& Gilkey, 2016). An array of studies documented that increased levels of regret is associated with increased levels of depression (e.g., Bruine de Bruin et al., 2016; Yu, Chen, Zhao, \& Yu, 2017). Another vein of regret studies focused on the role of regret in decision-making processes highlighting that regret is one of the vital emotions in the context of decision-making. One recent model concerning regret-decision making process is Decision Justification Theory that considered regret as two important components (DJT; Connolly \& Zeelenberg, 2002). Among the components, selfblame regret is strongly linked with mental health, particularly depression. In support, one crucial study demonstrated that currently depressed individuals reported greater levels of self-blame regret in response to hypothetical scenarios (Kraines, Krug \& Wells, 2017). Self-blame regret conveys the emotion when the individuals experience regret and blame themselves for the poorer decision (Wu \& Wang, 2017) and this component of regret focuses on self-responsibility for not selecting the better option (Nicolle, Bach, Frith \& Dolan, 2011). During such a pandemic, self-blame regret is off important to explore in terms of understanding the emotion and its relationship with mental health, specifically after normalization process began. Such time period is crucial as prior to the normalization process, prevention measures were maintained mainly by governments and home confinements and face mask wear were compulsory. Yet, normalization process eased some of the restrictions during the post peak pandemic and selfresponsibility in taking preventive measures became more important. In this regard, individuals are more prone to experience self-blame regret not to take enough cautions in case of contracting to the virus during post peak pandemic.

\section{Present Study}

As noted, research is scarce in terms of documenting the relationship between self-blame regret and mental health, specifically depression. To the best of the knowledge, there is only one study to report such link (Kraines, Krug \& Wells, 2017). Yet, more variables are needed to be examined in order to understand 
the emotion and its relationship with mental health. In this regard, fear of COVID-19 and perceived stress is potential mediators during the post peak pandemic. Thus, the main aim of this study was to test the relationship between self-blame regret and depression as a mental health indicator with a Turkish sample as mediated by individuals' levels of fear of COVID-19 and perceived stress. The hypotheses of the study included:

H7.Self-blame regret will be positively related to depression

H2.The relationship between self-blame regret and depression will be mediated by fear of COVID-19

H3. The relationship between self-blame regret and depression will be mediated by perceived stress

H4. The relationship between self-blame regret and depression will be serially mediated by fear of COVID19 and perceived stress.

\section{Methods}

\section{Participants}

Participants were recruited as a community sample of three hundred fifty two individuals ( $71 \%$ female and $29 \%$ males) and ranged between in age18 and $63(M=28.90 \pm 8.90)$. Their socioeconomic levels ranged from medium (70.5\%), high (15.1\%), very high (0.9\%) low (12.2\%) and very low (1.4\%).

Participants reported that the maximum number of people (friends and relatives) they lost to COVID-19 is 2 while acknowledged number of people tested positive is 38 .

\section{Measures}

Fear of COVID-19 Scale (FCV-19S).FCV-19S is comprised of seven items assessing the extent to which an individual fears COVID-19 (Ahorsu et al., 2020) and rated based on five-point Likert scale. Sum of the item scores provide the total score of the scale with higher scores indicative of higher levels of fear of COVID19. The psychometric properties of the Turkish form of the scale (Satici et al., 2020) have been widely supported and Cronbach's alpha reliability for the scale in this study was excellent $(a=.90)$

Perceived Stress Scale (PSS-10). PSS-10 is consisted of ten items measuring the extent to which situations in an individual life is perceived as stressful (Cohen et al., 1983) and rated based on five-point Likert scale. Sum of the item scores after reversing the negatively keyed items provide the total score of the scale with higher scores indicative of higher levels of perceived stress. The psychometric properties of the Turkish form of the scale (Kaya et al., 2019) have been widely supported and Cronbach's alpha reliability for the scale in this study was good $(a=.81)$

DASS-21 Depression Subscale (DASS-21). DASS-21 is comprised of twenty one items evaluating the severity and the frequency of the symptoms over the past week and rated based on four-point Likert scale (Lovibond \& Lovibond, 1995). The scale is consisted of three subscales of depression, anxiety and stress 
and the subscale scores are obtained via sum of the item scores of the relevant dimensions with higher scores indicative of higher levels of depression, anxiety and stress. The psychometric properties of the Turkish form of the scale (Yıldırım et al., 2018) have been widely supported and Cronbach's alpha reliability for the scale in this study was good $(a=.89)$

Self-blame Regret. Self-blame regret was evaluated with one item asking participants "How much would you blame yourself for not taking enough prevention measures if you catch the Coronavirus-19?" The item was rated based on 5 -point Likert scale ( $1=$ not at all, $5=$ extremely). Higher scores demonstrated higher levels of self-blame regret.

\section{Procedure}

Study procedure was approved by .......University Ethics Committee. Sample was reached through word of mouth and advertisement of the study on social media sites (Facebook, WhatsApp groups, etc.). Data collection started June 6, one day after normalization process was declared by the government.

Responses to the scales were collected via an online survey webpage, and the consent form to partake in the study was obtained via the first page of the online survey.

\section{Data Analyses}

In terms of main analyses, serial multiple mediation was performed with PROCESS macro for SPSS (Hayes, 2018) to test the mediation effects of how self-blame regret affects fear of COVID-19, how fear of COVID-19 affects perceived stress, and how perceived stress affects depression. Using 5000 bootstrap samples with $95 \%$ confidence intervals $(\mathrm{Cl})$, the paths that do not include zero was considered as the evidence of a significant indirect effect (Preacher \& Hayes, 2008).

\section{Results}

Table 1 demonstrates Cronbach's alpha reliability coefficients, the minimum, maximum, mean, standard deviation, skewness, kurtosis statistics for main study variables and Pearson Product-Moment correlations betweeen the variables. As shown in Table 1, no severe violations of normal hypotheses were encountered (e.g., skewness from 0.10 to 0.47 , kurtosis from -0.94 to 0.03 ) (West, Finch, \& Curran, 1995). Bivariate correlations between variables were computed and results demonstrated that self-blame regret was positively correlated with depression, fear of COVID-19 and perceived stress coefficients ranging between $r=.35$ and $r=.20$.

\section{Serial Multiple Mediation Analysis}

In order to test the mediating effect of fear of COVID-19 and perceived stress in the relationship between self-blame regret and depressive symptoms, a serial multiple mediation analysis was conducted. Results of the serial mediation analyses revealed positive direct effect of self-blame regret on depression supporting Hypothesis $1(\beta=.30, p<.001)$. Including mediators of fear of COVID-19 and perceived stress in the model, this coefficient was reduced though it was still significant $(\beta=.12, p<.05)$. Moreover, self- 
blame regret positively predicted both fear of COVID-19 $(\beta=.35, p<.001)$ and perceived stress $(\beta=.14, p<$ $.05)$. Figure 1 demonstrates the results of serial mediation estimations.

Serial mediation estimations also indicated significant indirect effect of self-blame regret on depression through fear of COVID-19 $(\beta=.05, \mathrm{SE}=.02,95 \% \mathrm{Cl}=[.017, .087])$ and perceived stress $(\beta=.09, \mathrm{SE}=.04$, $95 \% \mathrm{Cl}=[.013, .161])$ supporting Hypothesis 2 and 3 . Confirming hypothesis 4 , the indirect effect of selfblame regret on depression via fear of COVID-19 and perceived stress was tested and indirect effect was $0.04(\mathrm{SE}=.01,95 \% \mathrm{Cl}=[.013, .065])$. Table 2 shows standardized indirect effect of fear of COVID-19 and perceived stress on depression.

\section{Discussion}

Covid-19 pandemic caused strong negative emotions worldwide due to the strict measures to prevent the disease including compulsory home confinements, face mask wear, and social distancing. Yet, normalization process eased government-related restrictions during post peak pandemic that called upon self-responsibility to take preventive behaviors. Thus, individuals will be more prone to experience selfblame regret not to take enough cautions in case of contracting to the virus during such critical times. In this regard, current study sought to understand the role of self-blame regret on mental health issues during post peak pandemic and hypothesized that a) self-blame regret will be positively related to depression b) the relationship between self-blame regret and depression will be mediated by fear of COVID-19 c) the relationship between self-blame regret and depression will be mediated by perceived stress d) the relationship between self-blame regret and depression will be serially mediated by fear of COVID-19 and perceived stress.

In support with the first hypothesis, the findings demonstrated that self-blame regret was positively related to depression conveying that individuals high in levels of blaming themselves in case of virus contraction also demonstrated higher levels of depressive symptoms. Such results are in line with the previous literature. For instance, an array number of studies revealed that increased levels of regret is associated with elevated levels of depression (Chase et al., 2010; Lecci, Okun \& Karoly, 1994; Monroe, Skowronski, MacDonald \& Wood, 2005; Roese et al., 2009). In support and more importantly, one critical study revealed that clinically depressed individuals reported greater levels of self-blame regret compared to the healthy and previously depressed individuals (Kraines, Krug \& Wells, 2017).

Concerning the second hypothesis, the results revealed that fear of COVID-19 mediated in the relationship between self-blame regret and depression suggesting higher levels of self-blame regret contributes to increased levels of fear of COVID-19, which in turn contributes to elevated depressive symptoms. Essentially, fear of COVID-19 is a recent concept although its literature is rapidly expanding. Yet, no studies per see documented the link between self-blame regret and fear of COVID-19 and no study to support the findings of the current study. Despite, the finding is congruent in terms of the conceptual frameworks of the constructs. For instance, individuals who perceive that they will considerably blame themselves in case of catching the disease will also experience higher levels of fear concerning the virus. 
In a similar vein, empirical studies also supported that cancer patients' decisional regret scores predicted fear of recurrence of the disease (Maguire, Hanly, Drummond, Gavin \& Sharp, 2017). In regards with fear of COVID-19 and depressive symptoms, a growing body of pandemic research indicated that fear of COVID-19 impacts in depressive symptoms confirming the findings of the current study (Fitzpatrick, Harris \& Drawve, 2020).

Regarding the third hypothesis, the findings of this study reported that perceived stress mediated the link between self-blame regret and depression. The findings concur with the previous literature documenting the link between self-blame and psychological stress (Martin \& Dahlen, 2005; Straud \& McNaughtonCassill, 2019). Moreover, current pandemic studies also revealed that changes in stress levels was found correlated with changes in depressive symptoms (de Quervain et al., 2020).

In accordance with the main hypothesis, fear of COVID-19 and perceived stress mediated in the relationship between self-blame regret and depression. In line with this hypothesis, previous studies reported that fear of COVID-19 inflates perceived stress (Bakioğlu, Korkmaz \& Ercan, 2020); perceived stress (e.g., Zhang, Peters \& Chen, 2018) and particularly pandemic related stress contributes to elevated levels of depressive symptoms (Montano \& Acebes, 2020). Taking together, within the framework of all discussed findings, it can be concluded that self-blame regret impacts in depression through fear of COVID-19 and psychological stress.

There are central things to be highlighted in the present study. First, this is the first study to report the positive association between self-blame regret and fear of COVID-19. Essentially, the literature for selfblame regret is scarce though current study reported its vitality on one of the pandemic-related concepts, namely fear of COVID-19. Second, current study provided a comprehensive model to dismantle the relationships between emotion self-blame regret and depressive symptoms through fear of COVID-19 and perceived stress. Though, several limitations should be taken into consideration. Chief among these limitations, current study was cross-sectional and self-report in nature. Thus, future studies should employ other methods such as qualitative and longitudinal designs. Another limitation included the number of participants represented within the sample in terms of the gender. Despite recruitment procedure targeted a homogenous sample; number of females superseded the number of males within the sample. Thus, future studies should aim to include similar number of participants from both of the genders to support the generalizability of the results. Notwithstanding these limitations, this study illuminates the underlying mechanism behind the relationship between self-blame regret, fear of COVID19, perceived stress and depressive symptoms.

\section{Declarations}

All procedures followed were in accordance with the ethical standards of the responsible committee on human experimentation (institutional and national) and with the Helsinki Declaration of 1975, as revised in 2000 (5). Informed consent was obtained from all participants for being included in the study. 
Study procedure was approved by the University Ethics Comittee (Identifying information was ommitted for the sake of blind review procedure)

Funding: None to declare

Conflict of interest: None to declare

\section{References}

Bakioğlu, F., Korkmaz, O., \& Ercan, H. (2020). Fear of COVID-19 and Positivity: Mediating Role of Intolerance of Uncertainty, Depression, Anxiety, and Stress. International Journal of Mental Health and Addiction. Advance online publication. https://doi.org/10.1007/s11469-020-00331-y

Belen, H. 2020. Fear of COVID-19 and Mental Health: The Role of Mindfulness in During Time of Crisis. (Under Review)

Brewer, N. T., DeFrank, J. T., \& Gilkey, M. B. (2016). Anticipated regret and health behavior: A metaanalysis. Health Psychology, 35(11), 1264.

Brooks, S. K., Webster, R. K., Smith, L. E., Woodland, L., Wessely, S., Greenberg, N., \& Rubin, G. J. (2020). The psychological impact of quarantine and how to reduce it: rapid review of the evidence. The Lancet. Advance online publication. https://doi.org/10.1016/S0140-6736(20)30460-8

Bruine de Bruin, W., Dombrovski, A. Y., Parker, A. M., \& Szanto, K. (2016). Late-life depression, suicidal ideation, and attempted suicide: The role of individual differences in maximizing, regret, and negative decision outcomes. Journal of behavioral decision making, 29(4), 363-371.

Chase, H. W., Camille, N., Michael, A., Bullmore, E. T., Robbins, T. W., \& Sahakian, B. J. (2010). Regret and the negative evaluation of decision outcomes in major depression. Cognitive, Affective, \& Behavioral Neuroscience, 10(3), 406-413.

Choi, E. P. H., Hui, B. P. H., \& Wan, E. Y. F. (2020). Depression and anxiety in Hong Kong during COVID19. International journal of environmental research and public health, $17(10), 3740$.

Cohen, S., Janicki-Deverts, D., \& Miller, G. E. (2007). Psychological stress and disease. Jama, 298(14), 1685-1687.

Cohen, S., Kamarck, T., \& Mermelstein, R. (1983). A global measure of perceived stress. Journal of health and social behavior, 24(4), 385-396. Advance online publication. Doi: 10.2307/2136404

Connolly, T., \& Zeelenberg, M. (2002). Regret in decision making. Current directions in psychological science, 11(6), 212-216. 
de Quervain, D., Aerni, A., Amini, E., Bentz, D., Coynel, D., Gerhards, C., .. \& Schlitt, T. (2020). The Swiss Corona Stress Study. Advance online publication. Doi: 10.31219/osf.io/jqw6a

Du, J., Dong, L., Wang, T., Yuan, C., Fu, R., Zhang, L., Liu, B., Zhang, M., Yin, Y., Qin, J., Bouey, J., Zhao, M., \& $\mathrm{Li}, \mathrm{X}$. (2020). Psychological symptoms among frontline healthcare workers during COVID-19 outbreak in Wuhan. General hospital psychiatry, S0163-8343(20)30045-1. Advance online publication.

https://doi.org/10.1016/j.genhosppsych.2020.03.011

Fitzpatrick, K. M., Harris, C., \& Drawve, G. (2020). Living in the midst of fear: Depressive symptomatology among US adults during the COVID-19 pandemic. Depression and Anxiety. Advance online publication.https://doi.org/10.1002/da.23080

Gates, B. (2020). Responding to Covid-19-a once-in-a-century pandemic? New England Journal of Medicine, 382(18), 1677-1679.

Gotlib, I. H., Borchers, L. R., Chahal, R., Gifuni, A. J., \& Ho, T. (2020). Early Life Stress Predicts Depressive Symptoms in Adolescents During the COVID-19 Pandemic: The Mediating Role of Perceived Stress. Available at SSRN 3606441 or http://dx.doi.org/10.2139/ssrn.3606441

Harper, C. A., Satchell, L. P., Fido, D., \& Latzman, R. D. (2020). Functional fear predicts public health compliance in the COVID-19 pandemic. International journal of mental health and addiction. Advance online publication. https://doi.org/10.1007/s11469-020-00281-5

Kaya, C., Tansey, T. N., Melekoglu, M., Cakiroglu, O., \& Chan, F. (2019). Psychometric evaluation of Turkish version of the Perceived Stress Scale with Turkish college students. Journal of Mental Health, 28(2), 161167.

Kraines, M. A., Krug, C. P., \& Wells, T. T. (2017). Decision justification theory in depression: regret and selfblame. Cognitive Therapy and Research, 41(4), 556-561.

Lecci, L., Okun, M. A., \& Karoly, P. (1994). Life regrets and current goals as predictors of psychological adjustment. Journal of Personality and Social Psychology, 66(4), 731.

Limcaoco, R. S. G., Mateos, E. M., Fernandez, J. M., \& Roncero, C. (2020). Anxiety, worry and perceived stress in the world due to the COVID-19 pandemic, March 2020. Preliminary results. Advance online publication. https://doi.org/10.1101/2020.04.03.20043992

Lovibond, P. F., \& Lovibond, S. H. (1995). The structure of negative emotional states: Comparison of the Depression Anxiety Stress Scales (DASS) with the Beck Depression and Anxiety Inventories. Behaviour research and therapy, 33(3), 335-343.

Maguire, R., Hanly, P., Drummond, F. J., Gavin, A., \& Sharp, L. (2017). Regret and fear in prostate cancer: The relationship between treatment appraisals and fear of recurrence in prostate cancer survivors. Psycho-oncology, 26(11), 1825-1831. 
Martin, R. C., \& Dahlen, E. R. (2005). Cognitive emotion regulation in the prediction of depression, anxiety, stress, and anger. Personality and individual differences, 39(7), 1249-1260.

McAlonan, G. M., Lee, A. M., Cheung, V., Cheung, C., Tsang, K. W., Sham, P. C., ... \& Wong, J. G. (2007). Immediate and sustained psychological impact of an emerging infectious disease outbreak on health care workers. The Canadian Journal of Psychiatry, 52(4), 241-247.

Mo, Y., Deng, L., Zhang, L., Lang, Q., Liao, C., Wang, N., ... \& Huang, H. (2020). Work stress among Chinese nurses to support Wuhan in fighting against COVID-19 epidemic. Journal of nursing management. Advance online publication. DOI: 10.1111/jonm.13014

Monroe, M. R., Skowronski, J. J., MacDonald, W., \& Wood, S. E. (2005). The mildly depressed experience more post-decisional regret than the non-depressed. Journal of Social and Clinical Psychology, 24(5), 665-690.

Montano, R. L. T., \& Acebes, K. M. L. (2020). Covid stress predicts depression, anxiety and stress symptoms of Filipino respondents. International Journal of Research in Business and Social Science, 9(4), 78-103.

Nickell, L. A., Crighton, E. J., Tracy, C. S., Al-Enazy, H., Bolaji, Y., Hanjrah, S., ... \&Upshur, R. E. (2004). Psychosocial effects of SARS on hospital staff: survey of a large tertiary care institution. Cmaj, 170(5), 793-798.

Nicolle, A., Bach, D. R., Frith, C., \& Dolan, R. J. (2011). Amygdala involvement in self-blame regret. Social Neuroscience, 6(2), 178-189.

Pakpour, A. H., \& Griffiths, M. D. (2020). The fear of COVID-19 and its role in preventive behaviors. Journal of Concurrent Disorders. Advanced online publication.

Preacher, K. J., \& Hayes, A. F. (2008). Asymptotic and resampling strategies for assessing and comparing indirect effects in multiple mediator models. Behavior research methods, 40(3), 879-891.

Preti, E., Di Mattei, V., Perego, G., Ferrari, F., Mazzetti, M., Taranto, P., ... \& Calati, R. (2020). The Psychological Impact of Epidemic and Pandemic Outbreaks on Healthcare Workers: Rapid Review of the Evidence. Current Psychiatry Reports, 22(8), 1-22.

Reznik, A., Gritsenko, V., Konstantinov, V., Khamenka, N., \& Isralowitz, R. (2020). COVID-19 fear in Eastern Europe: Validation of the Fear of COVID-19 Scale. International journal of mental health and addiction. Advance online publication. https://doi.org/10.1007/s11469-020-00283-3

Roese, N. J., Epstude, K. A. I., Fessel, F., Morrison, M., Smallman, R., Summerville, A., ... \& Segerstrom, S. (2009). Repetitive regret, depression, and anxiety: Findings from a nationally representative survey. Journal of Social and Clinical Psychology, 28(6), 671-688. 
Satici, B., Gocet-Tekin, E., Deniz, M. E., \& Satici, S. A. (2020). Adaptation of the Fear of COVID-19 Scale: Its association with psychological distress and life satisfaction in Turkey. International Journal of Mental Health and Addiction. Advanced online publication. Advance online publication.

https://doi.org/10.1007/s11469-020-00294-0

Shepherd, L., \& O'Carroll, R. E. (2014). When do next-of-kin opt-in? Anticipated regret, affective attitudes and donating deceased family member's organs. Journal of health psychology, 19(12), 1508-1517.

Straud, C. L., \& McNaughton-Cassill, M. (2019). Self-blame and stress in undergraduate college students: The mediating role of proactive coping. Journal of American College Health, 67(4), 367-373.

Tsang, H. W., Scudds, R. J., \&Chan, E. Y. (2004). Psychosocial impact of SARS. Emerge Infect Disease, 10 (2004), 1326-27.

Wu, R., \& Wang, C. L. (2017). The asymmetric impact of other-blame regret versus self-blame regret on negative word of mouth. European Journal of Marketing.

Yıldırım, A., Boysan, M., \& Kefeli, M. C. (2018). Psychometric properties of the Turkish version of the Depression Anxiety Stress Scale-21 (DASS-21). British Journal of Guidance \& Counselling, 46(5), 582-595.

Yu, X., Chen, J., Zhao, S., \& Yu, X. (2017). Impact of rumination and regret on depression among new employees in China. Social Behavior and Personality: an international journal, 45(9), 1499-1509.

Zandifar, A., \& Badrfam, R. (2020). Iranian mental health during the COVID-19 epidemic. Asian journal of psychiatry. Advance online publication. https://doi.org/10.1016/j.ajp.2020.101990

Zhang, Y., Peters, A., \& Chen, G. (2018). Perceived stress mediates the associations between sleep quality and symptoms of anxiety and depression among college nursing students. International journal of nursing education scholarship, 15(1), 1-9. https://doi.org/10.1515/ijnes-2017-0020.

Zhang, Y., Wang, J., Zhao, J., Tanimoto, T., Ozaki, A., Crump, A., ... \& Jiang, Q. W. (2020). Association Between Quarantined Living Circumstances and Perceived Stress in Wuhan City During the COVID-19 Outbreak: A Rapid, Exploratory Cross-Sectional Study. Exploratory Cross-Sectional Study (3/16/2020). Available at SSRN: https://ssrn.com/abstract=3556642 or http://dx.doi.org/10.2139/ssrn.3556642

\section{Tables}


Table 1. Descriptive statistics and intercorrelations between main study variables

\begin{tabular}{lccccccccccc}
\hline & 1 & 2 & 3 & 4 & $\alpha$ & Min & Max & M & SD & Skew & Kurt \\
\hline 1.Self-blame R & 1 & & & & - & 1.00 & 5.00 & 2.92 & 1.35 & 0.14 & -0.94 \\
2.Depression & $.30^{* *}$ & 1 & & & .89 & 0.00 & 21.00 & 9.68 & 5.76 & 0.10 & -0.92 \\
3.Fear of COVID & $.35^{* *}$ & $.32^{* *}$ & 1 & & .90 & 7.00 & 35.00 & 17.42 & 6.77 & 0.47 & -0.41 \\
4.Per. Stress & $.20^{* *}$ & $.68^{* *}$ & $.21^{* *}$ & 1 & .81 & 14.00 & 48.00 & 28.67 & 6.69 & 0.24 & 0.03 \\
\hline
\end{tabular}

Note. ${ }^{* *} . p<.001$

Table 2. Standardized indirect effect of fear of COVID-19 and perceived stress on depression

\begin{tabular}{|c|c|c|c|}
\hline \multirow[t]{2}{*}{ Path } & \multirow[t]{2}{*}{ Coefficient } & \multicolumn{2}{|c|}{$95 \% \mathrm{CI}$} \\
\hline & & UL & LL \\
\hline Self-blame regret $\square$ Fear of Covid-19₫ Depression & .05 & .017 & .087 \\
\hline Self-blame regret $\square$ Perceived Stress $\square$ Depression & .09 & .013 & 161 \\
\hline Self-blame regret $\square$ Fear of Covid-19₫ Perceived & .04 & .013 & .065 \\
\hline Stress $\square$ Depression & & & \\
\hline Total Indirect Effect & 17 & .093 & 253 \\
\hline
\end{tabular}

Note. $\mathrm{CI}=$ Confidence Interval, $\mathrm{LL}=$ Lower Limit, $\mathrm{UL}=$ Upper

Limit

\section{Figures}

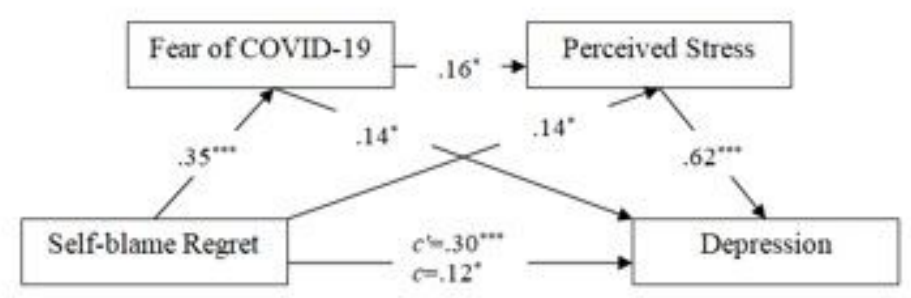

\section{Figure 1}

Serial multiple mediational model. Path coefficients were standardised; ${ }^{*} p<.05,{ }^{\star \star} p<.01,{ }^{\star \star *} \mathrm{p}<.001$. 